\title{
Matematización y modelización: experiencias y saberes. Una propuesta de aula*
}

\author{
Andrés Mauricio Martínez Novoa \\ Estudiante de la Licenciatura en Educación Básica con Énfasis en \\ Matemáticas de la Universidad Distrital Francisco José de Caldas. \\ Correo electrónico: martinez9420@hotmail.com
}

\author{
Juan Camilo Cobos Caicedo \\ Estudiante de la Licenciatura en Educación Básica con Énfasis en \\ Matemáticas de la Universidad Distrital Francisco José de Caldas. \\ Correo electrónico: camilo.cobos91@gmail.com
}

\author{
Elizabeth Torres Puentes \\ Magíster en Educación. Estudiante de Doctorado en Educación. \\ Docente de la Licenciatura en Educación Básica con Énfasis en \\ Matemáticas de la Universidad Distrital Francisco José de Caldas. \\ Correo electrónico: elizatorrespuentes@gmail.com
}

\section{Cómo citar este artículo:}

Martínez, A. M., Cobos, J. C., y Torres, E. (2015). Matematización y modelización: experiencias y saberes. Una propuesta de aula. Espiral. Revista de Docencia e Investigación, 5(2), 9-22.

\section{Resumen}

Este artículo de investigación presenta una secuencia didáctica enmarcada en el espacio de formación: Ambientes y Mediaciones del Aula, de la Licenciatura en Educación Básica con Énfasis en Matemáticas de la Universidad Distrital Francisco José de Caldas. Dicha secuencia se aplicó y validó en la práctica docente que se realizó en un colegio Distrital de la ciudad de Bogotá, y tuvo como objetivo fundamental desarrollar el concepto de magnitud-longitud, partiendo de las relaciones que se presentan entre la matematización y la modelización, para lo cual se usó el trabajo de investigación sobre matematización de Freudenthal (1968).

La secuencia se basó en una serie de actividades realizadas en grado segundo de primaria, tomando como metodología de gestión el modelo DECA (1992). Los resultados más relevantes se relacionan con el cómo los estudiantes a través de situaciones matematizables puestas en un contexto concreto llegan a la modelización del metro, decímetro y centímetro, como la unidad de medida estandarizada.

Palabras clave: Matematización, modelización, magnitud-longitud, modelo matemático, unidad de medida.

\section{Abstract}

This article shows a didactic sequence framed in space environments and mediation training classroom of the Degree in Basic Education with emphasis on Mathematics, University Francisco José de Caldas. This sequence was applied and validated in teaching practice which took place in a District school in the city of Bogota, and had as main objective to develop the concept of scalelength, based on the relationships that exist between the mathematization and modeling, for which research work mathematisation Freudenthal (1968) was used.

The sequence is based on a series of activities in second grade, building management methodology DECA model (1992). The most relevant results are related to how students through situations matematizables placed in a specific context, modeling reach metro, decimeter and centimeter as the unit of standard measure.

Keywords: Mathematization, modeling, scalelength, mathematical model, unit of measure.

\section{Resumo}

Este artigo mostra uma seqüência didática enquadrado em ambientes espaciais e sala de aula de treinamento de mediação da Licenciatura em Educação Básica

* Artículo de investigación producto del desarrollo del espacio de formación: Ambientes y Mediaciones en el Aula, del proyecto curricular Licenciatura en Educación Básica con Énfasis en Matemáticas de la Universidad Distrital, Facultad de Educación y el Centro de Investigaciones y Desarrollo Científico. 
com ênfase em Matemática da Universidade Francisco José de Caldas. Esta sequência foi aplicado e validado na prática docente que teve lugar em uma escola District, na cidade de Bogotá, e teve como objetivo principal desenvolver o conceito de escala de comprimento, com base nas relações que existem entre a matematização e modelagem, para o qual foi utilizado trabalho de investigação matematização Freudenthal (1968).

A sequência é baseado em uma série de atividades em segundo grau, a construção de modelo DECA metodologia de gestão (1992). Os resultados mais relevantes estão relacionados com a forma como os alunos através de situações matematizables colocado em um contexto específico, modelagem chegar metro, decímetro e centímetros como unidade de medida padrão.

Palavras-chave: Matematização, modelagem, a escala de comprimento, modelo matemático, unidade de medida.

\section{Introducción}

Este artículo exhibe una secuencia didáctica desarrollada en el marco del curso Ambientes y Mediaciones del Aula'. Dicha secuencia tiene como base fundamental la práctica educativa realizada en una institución educativa distrital de la ciudad de Bogotá, y su propósito fue el desarrollo del concepto magnitud-longitud. Este concepto fue construido partiendo de las relaciones que hay entre la modelización y matematización. En la práctica docente se evidenciaron ciertas tensiones que surgen entre dichas relaciones para el desarrollo del concepto magnitud-longitud en estudiantes de grado segundo.

La primera tensión se refiere al poco desarrollo del vínculo entre las situaciones matematizables y la modelización. Un ejemplo de lo anterior es que los niños en una etapa inicial dependen del uso de la regla como un instrumento de medida que interviene, según ellos, en la solución de todas las situaciones que involucran la noción de la magnitud longitud.

1 Este espacio de formación hace parte del currículo de la Licenciatura en Educación Básica con Énfasis en Matemáticas (LEBEM) de la Universidad Distrital Francisco José de Caldas.
Para Freudenthal (1971) la matemática es una actividad humana y como actividad trata de solucionar problemas o reconocerlos en un contexto determinado. Según el autor, la matemática es también una actividad de organización de la disciplina misma. Esta actividad puede estar dirigida a considerar una parte de la realidad que llama a ser organizada de acuerdo con patrones matemáticos.

El autor alemán reconoce que matematizar es la búsqueda de lo esencial en la realidad². Expresar esa esencia implica resolver situaciones problemas (procedimientos, algoritmos, formulaciones, simbolizaciones y sistemas axiomáticos), que se desarrollan durante un proceso en el que los alumnos reinventan ideas y utilizan herramientas matemáticas, para organizar o estructurar situaciones problemáticas en interacción con sus compañeros y bajo la guía del profesor. Sin embargo, en el desarrollo de la propuesta de aula se encontró que los estudiantes no reinventan el concepto de magnitud-longitud, ya que este no se contextualiza, es decir, la idea matemática no nace de la necesidad de resolver una situación que se presente desde la realidad.

Partiendo de las investigaciones de Kieran (1992), Treffers y Goffree (1985); Treffers (1991), Gravemeijer y Teruel (2000) se entiende que la modelización, es reunir las estructuras o relaciones que hay en una situación problema, que a su vez está puesta en el mundo real. Por su parte Blomhøj (2004) afirman que la modelización matemática es capaz de llevar a cabo en forma autónoma y consiente todos los aspectos de un proceso de modelización en un contexto dado, por ende, los estudiantes de grado segundo tienen la noción de medida a partir de situaciones que no están contextualizadas en la realidad, porque la aparición de la medida y con ella, la del sistema decimal es algo espontáneo y carece de significado, es por eso que no se pueden hacer modelizaciones del mundo real si desde un

2 Llamaremos realidad como dice Freudenthal (1991, p. 17) aquello que el sentido común experimenta como real a un cierto escenario. 


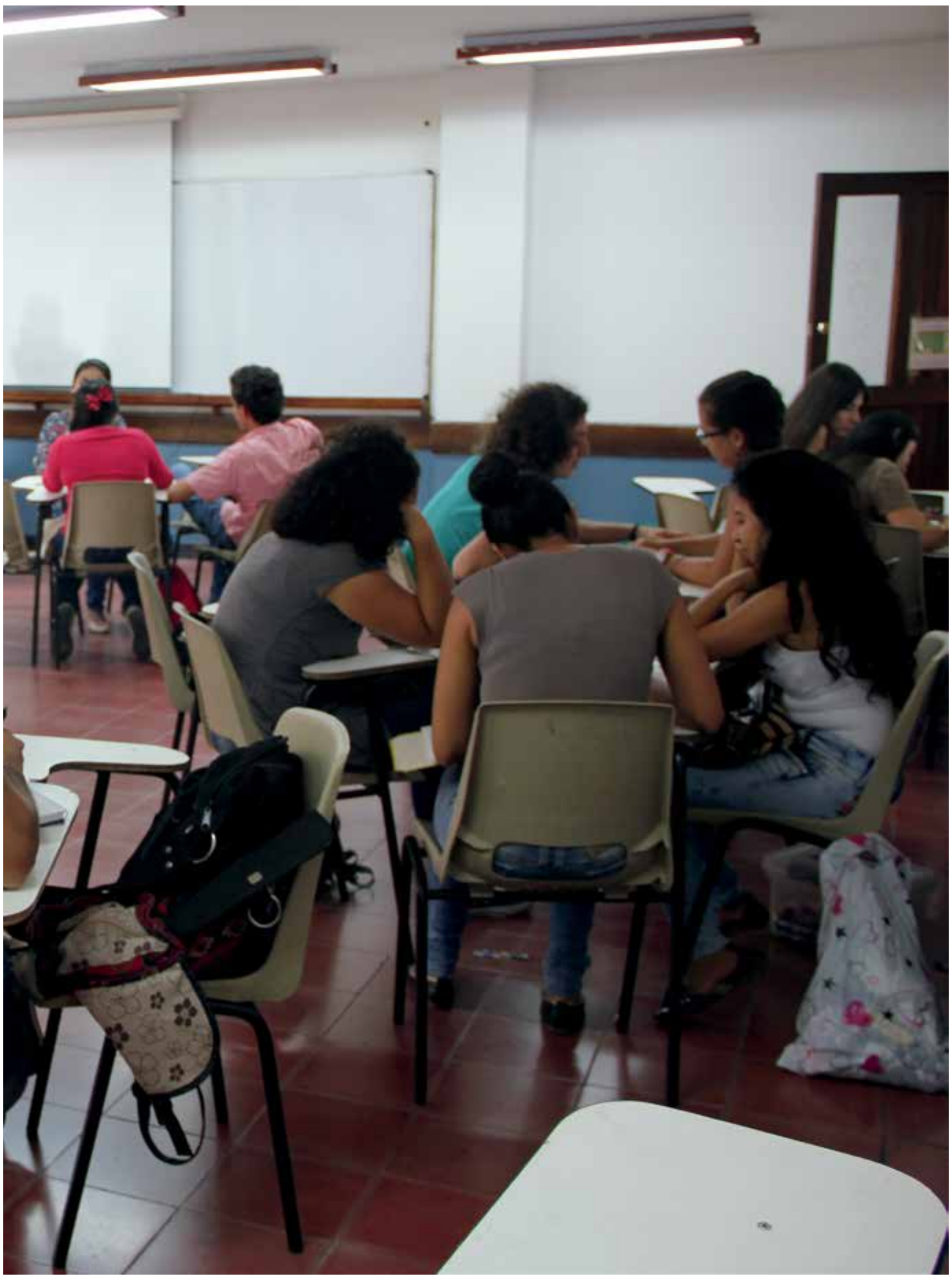


principio no se ha realizado una matematización del concepto propiamente dicho.

La segunda tensión reconoce que la relación entre modelización y matematización no es potenciada en el aula de segundo grado, es decir, el paso que se da entre las estructuras que nacen a partir de las situaciones matematizables y la creación de modelos que reúnan tales estructuras no son fortalecidas, y en la mayoría de casos esas actividades que podrían potenciar tal relación, se resumen a la enseñanza de la medición de objetos con instrumentos como la regla o el metro, por ello se pierden ciertas conceptualizaciones que priman en matematizar cómo reconocer el sistema decimal que se usa en el sistema métrico.

De tal manera que las estructuras que se podrían generar de las situaciones quedan relegadas al hecho de aprender cuántos centímetros tiene una regla, o conocer los múltiplos y submúltiplos de tal unidad, resultados que conceptualmente están resumidos en un modelo que no fue el resultado de todo un proceso, como sí lo fue la actividad de medir en lo que conocemos de la evolución del hombre.

Partiendo de las tensiones encontradas, se expone en este artículo una propuesta de aula bajo las ideas de la matematización y modelización, para potenciar la construcción del concepto magnitud-longitud y dotar de significado el acto de medir. También se mostrarán los resultados durante el proceso de aprendizaje, para poder contrastar la enseñanza tradicional de los instrumentos de medida y las potencialidades de las actividades que se realizaron en la práctica docente.

\section{Metodología}

La secuencia didáctica que se exhibe en este artículo está enmarcada en los conceptos de matematización, modelos matemáticos, modelización matemática, estadios principales de la constitución de la magnitud-longitud, la percepción y constitución de la unidad de medida.
Como se ha descrito las relaciones que se dan entre la matematización y la modelación nos permite el desarrollo de dicho concepto, pero esas relaciones son las que no se están potenciando dentro del ambiente de aprendizaje, por lo tanto, la enseñanza-aprendizaje está desprovista de significado porque se llega a una construcción del concepto carente de significado en la vida real o situaciones concretas. La idea principal de Freudenthal (1971) es que las matemáticas deben ser concebidas como una actividad humana. Para este autor la matemática es la actividad de resolver problemas y buscar problemas $y$, en términos más generales, la actividad de organizar la disciplina a partir de la realidad o de la matemática misma, a esto último le dio el nombre de Matematización. Esta interpretación de las matemáticas es la que se utilizará para explicitar en este artículo la secuencia didáctica, porque en palabras más sencillas partiendo de situaciones relacionadas con la realidad los niños pueden estructurar en lenguaje matemático el concepto de magnitud-longitud, es decir, matematizar la medida.

Por su parte, dice Niss (1989) que la modelización matemática se define como el arte de aplicar las matemáticas a la vida real, de tal manera que la matematización sería la metamorfosis de la realidad en lenguaje matemático, por ende la modelización sería adaptar las estructuras matemáticas a la realidad.

La relación que hay entre matematización y modelización es estrecha. Se accede a la matematización por medio de la realidad arribando a las estructuras que están presentes en la existencialidad. En el planteamiento de la modelización matemática está implícito el proceso de matematización.

En 1991 Freudenthal expresó que existen dos tipos de matematización: "la matematización horizontal, implica ir del mundo real al mundo de los símbolos, mientras que la matematización vertical, significa desenvolverse en el mundo de los símbolos" (pp. 41-42). Durante 
los procesos de modelización matemática se utilizan ambos tipos de matematización: en un primer momento la matematización horizontal, ajustando el problema real a un modelo matemático, para después, en el proceso de matematización vertical, manipularlo y llegar a su resolución.

Explicitando las relaciones que hay entre esos dos conceptos tan relevantes, es importante contrastar con la secuencia didáctica. Existen diferentes etapas que utilizadas en la enseñanza permiten erigir una ruta de aprendizaje mediante los cuales se construye la magnitud-longitud. Para emprender esa cimentación es ineludible comenzar por los estadios principales en el desarrollo de la magnitud-longitud. Chamorro y Belmonte (1994) suponen cuatro estadios:

1. Consideración y percepción de una magnitud como una propiedad que posee una colección de objetos, sin tener en cuenta otras propiedades que puedan presentar tales objetos.

2. Conservación de una magnitud, estadio que se considerará superado en el momento en que el alumno haya adquirido la idea de que, aunque el objeto cambie de posición, forma, tamaño o alguna otra propiedad, sin embargo hay algo que permanece constante: ese algo es, precisamente aquella magnitud respecto a la cual pretendemos que el niño sea conservador.

3. Ordenación respecto a una magnitud dada: solo cuando el alumno sea capaz de ordenar objetos teniendo en cuenta únicamente la magnitud considerada.

4. El último tramo coincide con el momento en que el niño sabe establecer una relación entre la magnitud y el número, momento en que es capaz de medir.

Cuando el niño supera estos estadios se evidencian los primeros criterios de medida: la medida espontánea. Esta idea basada en Piaget, desde un punto de vista psicológico, trata de esclarecer, en lo posible, los mecanismos que intervienen en la construcción de la medida, para sentar unas bases sólidas que permitan una adquisición adecuada de la idea de medida en cualquier magnitud particular considerada. A través de los estadios piagetianos, Chamorro y Belmonte (1994) explicitan dos momentos en el desarrollo evolutivo de la idea de medida:

- Comparaciones perceptivas: para medir, el niño utiliza al principio una medida perceptiva. Consiste en medir a partir de impresiones sensoriales, antes de adoptar un útil de medida móvil.

- Etapas principales: se reconocen los estadios piagetianos sobre el desarrollo evolutivo de la idea de medida:

- Estadio de la comparación perceptiva directa entre dos objetos, sin recurrir a ninguna medida común ni a ningún otro desplazamiento. En este estadio se pueden distinguir dos fases: en la primera, la estimación es completamente directa, de forma que, por ejemplo, si se pide a un niño que construya una torre igual a otra, suele hacerlo de una forma sumaria y sincrética. En la segunda, las estimaciones ya son mucho más analíticas, ya que no solo utiliza el transporte visual, sino también los transportes manual y corporal y, por tanto, pasa de una forma primitiva de medición a formas más ligadas a lo que es realmente medir.

- Estadio caracterizado por el desplazamiento de objetos de uno de los dos términos de la comparación perceptiva directa, o por la intención de un término medio precedente de la medida común, pero sin hacerse operatoria todavía la transitividad. En este estadio se pueden distinguir dos etapas: 
la del transporte manual, consistente en aproximar los objetos que tratamos de comparar, con lo que la estimación visual no realiza con una distancia apreciable de por medio, sino entre los objetos pegados entre sí prácticamente. El alumno se sirve de un término medio, pero que no es todavía una medida común e independiente, ya que normalmente utiliza partes de su propio cuerpo: dedos, palmas, pies, entre otros. Con ese término medio empieza a comparar los dos objetos enfrentados, lo cual supone un primer avance verdaderamente importante hacia la construcción de la idea de unidad de medida (pp. 18-19).

Durante el desarrollo de las situaciones matematizables también se trabajaron diferentes construcciones de la unidad planteadas por Chamorro y Belmonte (1994):

a) Ausencia de unidad. La primera medida infantil es puramente visual y comparativa. Así, se pueden comparar dos objetos directamente entre sí, pero se complica la comparación si introducimos un tercer objeto, $y$, aunque se puedan dar ciertos avances en la comparación de medidas de los tres objetos en una magnitud determinada, ello no supone nunca la idea ni la utilización de una unidad de medida.

b) Unidad objetal. Es una unidad ligada a un solo objeto y claramente relacionada con lo que debe medirse, formando incluso parte de la misma función que tiene el objeto que ha de ser medido. Está tan ligada a éste que, ante dos medidas presuntamente iguales, el niño puede errar su estimación, suponiendo que se rompa esa relación existente entre esa unidad y el objeto por medir. Sin embargo, esa falta de independencia no le impedirá utilizarla como una especie de unidad para la medida de otros objetos, una vez que ha sido usada en su primera función. c. Unidad situacional. Unidad que depende todavía fuertemente del objeto por medir, pero que cambia o puede cambiar de un objeto a otro, siempre que para cada uno se realice la medición y se conserve una cierta relación, al menos en orden de magnitud, entre las unidades respectivas. Esta relación tendrá que ver con la que existe entre los objetos para medir, dentro de una magnitud determinada.

d. Unidad figural. Aquí, la unidad por construir va perdiendo toda relación con el objeto a medir, incluso en el orden de magnitud, permaneciendo, eso sí, una cierta tendencia a medir objetos grandes con unidades grandes, y objetos pequeños con pequeñas unidades. La adecuación de la unidad a la magnitud de lo medible -condición no indispensablehace que el avance hacia la consecución de la unidad sea importante.

e. Unidad propiamente dicha. La unidad se ve totalmente libre de la figura u objeto considerado, tanto en forma como en tamaño, y es cuando se consigue una unidad propiamente interfigural, la misma para todas las figuras u objetos. Llegados a este extremo se tendrá como resultado de la medida un número y ambas nociones medida y número se enriquecen entre sí al tratar de medir con una misma unidad objetos de diferente tamaño, forma, textura o densidad (pp. 21-22).

Se ha ido pasando de una unidad, en principio ligada totalmente al objeto a medir (intraobjeto), a una unidad que no depende en absoluto de dicho objeto (inter-objeto), es decir, se considera la necesidad de medir la longitud de algunos objetos haciendo uso de la medida estandarizada (metro, decímetro, centímetro). En la obtención de esa unidad perfecta de medida no se han perdido las características 


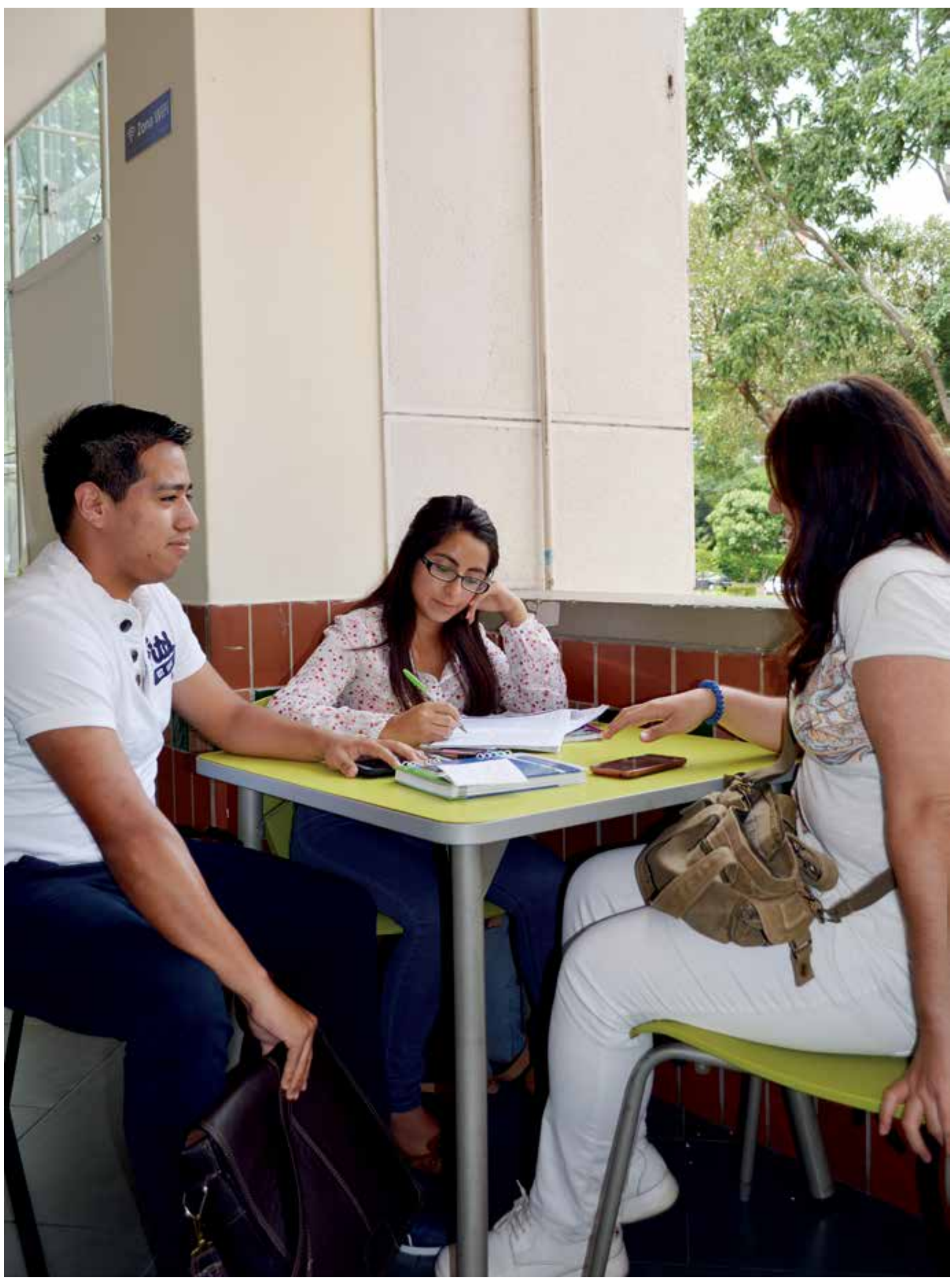


primitivas para las que sirven la medida dentro de una magnitud, aunque tampoco las deja en el mismo estado en que se habían tomado. Se va construyendo una unidad cada vez más perfecta y desligada de lo que se ha de medir, y que hace que la medida de cualquier objeto vaya evolucionando hacia una mayor facilidad, perfeccionando al mismo tiempo los métodos o procedimientos de medida.

Para desarrollar la secuencia didáctica se usó el estudio de caso, pues se tomó la experiencia del diseño, gestión y evaluación de una secuencia didáctica para estudiantes del grado segundo de la básica primaria.
Como instrumentos para recabar la información, se usó la secuencia didáctica propiamente y se aplicaron dos entrevistas semiestructuradas a una docente de la Universidad Distrital Francisco José de Caldas, y a una estudiante egresada de la Licenciatura en Educación Básica con Énfasis en Matemáticas, respectivamente. Los resultados de estas entrevistas permitieron situar la importancia de la matematización y la modelización en el aprendizaje de la medida en el contexto escolar.

A continuación se transcriben apartes de las entrevistas, que son valiosos para el fin de este artículo:
Pregunta Profesora formadora de formadores

Un modelo matemático es un recurso que permite dar solución

¿Qué entiende por modelo matemático? a cierto tipo de situaciones que pueden estar dentro de un contexto matemático o dentro de un contexto real.
Egresada de la Licenciatura

Modelar matemáticamente para mí es tomar un objeto de la naturaleza o un fenómeno que sucede en nuestro diario vivir $y$ ponerlo a funcionar de manera matemática, mirar sus cualidades, sus posibles formas de comportarse en el mundo a partir de la matemática.
Matematizar es lograr llevar al lenguaje matemático las situaciones reales o concretas.
Matematización para mi es crear el modelo matemático, una función o algo que describa un fenómeno que sucede, entonces a partir de ciertas características que se puedan observar en cierto fenómeno se puede construir una función o algo que describa ese fenómeno y permita saber el comportamiento que va a tener en algún momento específico.
Ahora bien, como observamos en las respuestas, encontramos que partiendo de ideas familiares de las dos entrevistadas, acerca de matematización y modelación, además de su experiencia en la práctica docente, resultó conveniente para ambas personas desarrollar el concepto de magnitud-longitud a partir de las actividades que potencien la relación entre matematizar y modelar.
A continuación se presenta la malla de planeación de la secuencia de actividades, la cual está enmarcada en el modelo del Grupo DECA. Este modelo propone las fases de desarrollo para las actividades que se clasifican de la siguiente forma (Lurdy, Guerrero, Sánchez, 2005): 


\section{MODELO DE ACTIVIDADES GRUPO DECA}

\begin{tabular}{|c|c|}
\hline Activdades & Sirven para que el estudiante \\
\hline 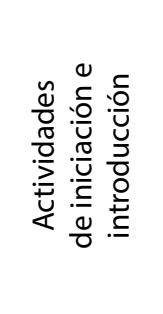 & $\begin{array}{l}\text { - } \quad \text { Explicite y exteriorice sus ideas previas sobre los contenidos que se van a tratar en la SD. } \\
\text { - } \quad \text { Compruebe la necesidad de trabajar esos contenidos. } \\
\text { - } \quad \text { Se predisponga favorablemente para afrontar el desarrollo de la UD con una actitud positiva. } \\
\text { - } \quad \text { Compruebe que sus conocimientos y estructuras conceptuales anteriores no son los más adecuados para } \\
\text { - Caiga en un conflicto interno cognitivo que le fuerce a un cambio en sus esquemas de conocimiento. }\end{array}$ \\
\hline 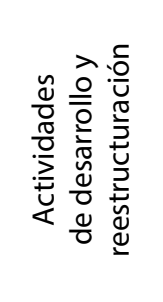 & $\begin{array}{l}\text { - Tomar contacto, practicar y asimilar los nuevos contenidos. } \\
\text { - } \quad \text { Reflexionar sobre su utilidad a la hora de enfrentarse a nuevas situaciones. } \\
\text { - } \text { pomparar con los conocimientos anteriores, comprobar sus ventajas e incorporarlos a su experiencia } \\
\text { - Producir el cambio deseado en sus esquemas mentales, como consecuencia de la superación del conflicto } \\
\text { cognitivo aparecido con las actividades de iniciación. }\end{array}$ \\
\hline
\end{tabular}

- Aplicar a otras situaciones los nuevos conocimientos adquiridos.

$\frac{0}{0}>$ 은 Reflexionar sobre las características esenciales de esos contenidos.

政

- Facilitar el trabajo en pequeñas investigaciones, relacionadas con los contenidos trabajados.

- $\quad$ Proponer situaciones de carácter opcional, dependiendo del nivel de dificultad y de la situación personal de cada alumno/na.

- $\quad$ Conocer el grado de los aprendizajes que los alumnos han adquirido.

- Permitir que los alumnos conozcan la utilidad del trabajo realizado y lo que han aprendido.

- Verbalizar algunos aprendizajes.

- Detectar errores, inexactitudes, fallos.

- Permitir reforzar aprendizajes.

\section{A continuación presentamos la secuencia de actividades aplicada}

\begin{tabular}{|c|c|c|}
\hline $\begin{array}{c}\text { Tipo de } \\
\text { actividad }\end{array}$ & $\begin{array}{c}\text { Nombre de la } \\
\text { actividad }\end{array}$ & Criterios de matematización y modelación \\
\hline \multirow{5}{*}{ 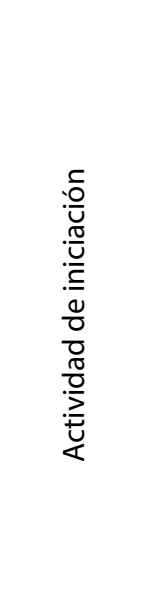 } & \multirow{3}{*}{ Cerebremos } & $\begin{array}{l}\text { Criterio } 1 . \\
\text { Los estudiantes observan en los objetos dimensiones, que pueden ser caracterizadas con } \\
\text { palabras como es alto, largo, ancho, corto entre otras. }\end{array}$ \\
\hline & & \\
\hline & & $\begin{array}{l}\text { Criterio } 2 . \\
\text { Los estudiantes relacionan las dimensiones de los objetos como atributos medibles, es decir, } \\
\text { cosas que se pueden medir. }\end{array}$ \\
\hline & \multirow[b]{2}{*}{ Torres creativas } & $\begin{array}{l}\text { Criterio } 1 . \\
\text { Los estudiantes construyen a partir de fichas una torre, en la cual identifican una dimensión } \\
\text { precisa (altura). }\end{array}$ \\
\hline & & $\begin{array}{l}\text { Criterio } 2 . \\
\text { Se identifican relaciones entre el cambio de la torre, es decir, aunque traslade la torre de lugar, } \\
\text { o la gire con respecto a un eje, las fichas en ella no cambian de cantidad o numerosidad, es } \\
\text { decir conserva su magnitud en cuanto a fichas a pesar de los movimientos. }\end{array}$ \\
\hline
\end{tabular}




\begin{tabular}{|c|c|c|}
\hline $\begin{array}{l}\text { Tipo de } \\
\text { actividad }\end{array}$ & $\begin{array}{l}\text { Nombre de la } \\
\text { actividad }\end{array}$ & Criterios de matematización y modelación \\
\hline 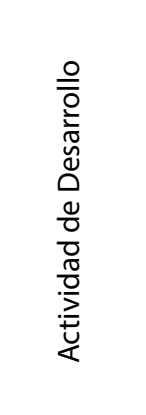 & $\begin{array}{l}\text { ¿Qué tan alto eres, } \\
\text { que tan cerca estas? }\end{array}$ & $\begin{array}{l}\text { Criterio } 1 . \\
\text { Los estudiantes comparan su propia estatura con la de sus compañeros, y encuentran } \\
\text { maneras de ordenarse entre ellos con respecto a esta dimensión. } \\
\text { Criterio } 2 \text {. } \\
\text { Los estudiantes reconocen qué tan cerca están los objetos o qué tan lejos. } \\
\text { Criterio } 3 . \\
\text { Los estudiantes diferencian entre la dimensión de su altura y la distancia entre objetos, } \\
\text { reconocen que las alturas son la dimensión entre cabeza y pies, y distancia el espacio vacío } \\
\text { entre dos objetos. }\end{array}$ \\
\hline
\end{tabular}

\section{Criterio 1.}

\begin{tabular}{|c|c|}
\hline 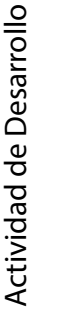 & Manos y pies \\
\hline
\end{tabular}

Los estudiantes encuentran instrumentos de medida, como lo son sus manos, pies, brazos, entre otros.

\section{Criterio 2.}

Los estudiantes miden con sus manos o pies las dimensiones o distancias, relacionando la numerosidad con la unidad con la que miden.

\section{Criterio 3.}

Los estudiantes encuentra conveniente medir con partes del cuerpo, y reconocen que los resultados serán diferentes con respecto a la persona que mida.

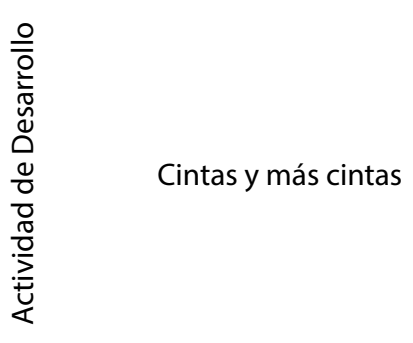

\section{Criterio 1.}

Los estudiantes resuelven la situación de tener medidas diferentes, recurriendo a medir con objetos iguales, y tan solo miden como lo hacían con sus manos, pero ahora con unidades convencionales (cintas).

\section{Criterio 2.}

Los estudiantes reconocen que las unidades iguales permiten generalizar los resultados, y que con relación a la dimensión del objeto, es preferible medirlo con unidades según la dimensión.

\section{Criterio 1.}

Los estudiantes establecen las relaciones matemáticas entre las unidades al medir, y la numerosidad, además de reconocer que los patrones de medida pueden generar múltiplos de la unidad inicial, es decir, encuentran conveniente estandarizar las unidades a través de equivalencias, ejemplo de esto " 5 cintas de color amarillo, son iguales a una azul" por ende Armando conceptos encuentran en las cintas unidades más factibles para medir cosas más grandes.

\section{Criterio 2.}

Los estudiantes matematizan las relaciones entre las cintas y la actividad de medir, es decir, encuentran que al medir dimensiones u objetos, consiste en reiterar un patrón, y de acuerdo con la numerosidad de unidades, se encuentra la medida de algún objeto.

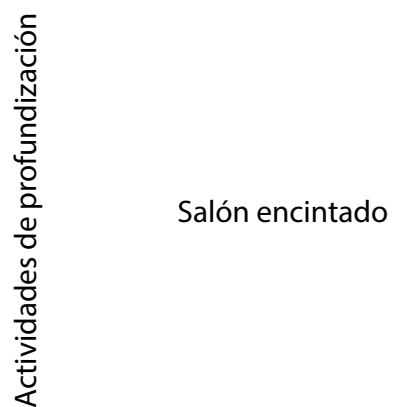

\section{Criterio 1.}

Los estudiantes reconocen los múltiplos de diez de las cintas dadas, y aun sin modelar al metro o la regla en centímetros, los niños miden partes de su alrededor con las cintas y son capaces de expresar la medida en términos de la unidad dispuesta en cintas o partes más pequeñas de la cinta más grande.

\section{Criterio 2.}

Los estudiantes expresan con facilidad las dimensiones o distancias, además de medir otros objetos, y reconocer a la cinta como un modelo ideal para medir por la facilidad de uso en la actividad. 
¿Qué tan alto eres, que tan cerca estas?

\section{Criterio 1.}

Los estudiantes comparan su propia estatura con la de sus compañeros.

\section{Criterio 2.}

Los estudiantes reconocen la distancia que separa a las dos torres.

\section{Criterio 3.}

Los estudiantes diferencian entre la dimensión de su altura dibujada en el cuaderno, y la distancia entre las torres.

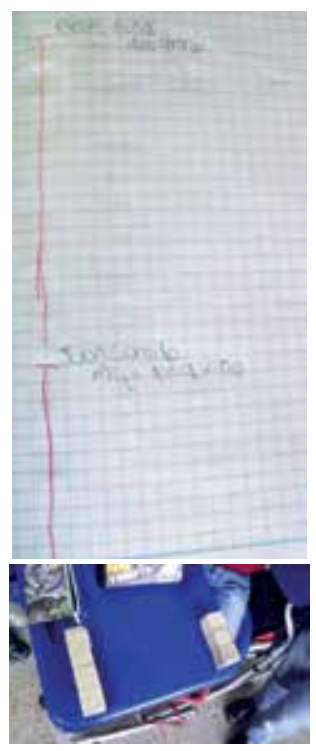

Manos y pies

\section{Criterio 1.}

Los estudiantes encuentran maneras de medir con sus pies o sus manos como se puede ver en las imágenes.

\section{Criterio 2.}

Los estudiantes comprenden que las medidas no serán iguales por ser las partes del cuerpo de diferentes personas.
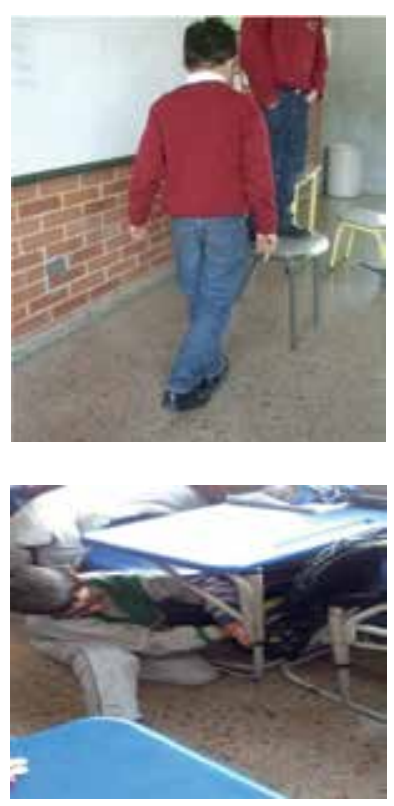

Cintas y más cintas

\section{Criterio 2.}

Los estudiantes reconocen que las unidades iguales permiten generalizar los resultados, y que con relación a la dimensión del objeto, es preferible medirlo con unidades según lo grande que resulte ser.

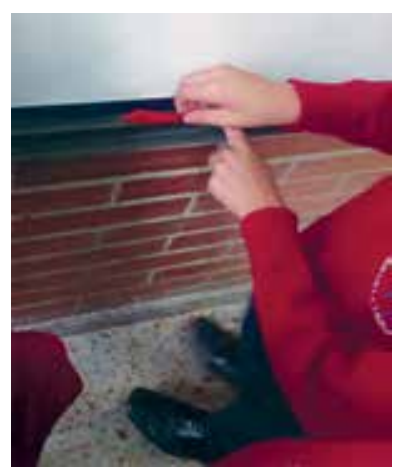


Evidencia

Criterio en relación con el criterio evaluado

Criterio 1.

Los estudiantes establecen las relaciones matemáticas entre las unidades al medir, y la numerosidad, además de reconocer que los patrones de medida pueden generar múltiplos de la unidad inicial, es decir, encuentran conveniente estandarizar las unidades a través de equivalencias, ejemplo de esto " 5 cintas de color amarillo, son iguales a una azul" por ende, encuentran en las cintas unidades más factibles para medir cosas más grandes.

\section{Criterio 2.}

Los estudiantes matematizan las relaciones entre las cintas y la actividad de medir, es decir, encuentran que al medir dimensiones u objetos, consiste en reiterar un patrón, y de acuerdo con la numerosidad de unidades, se encuentra la medida de algún objeto.

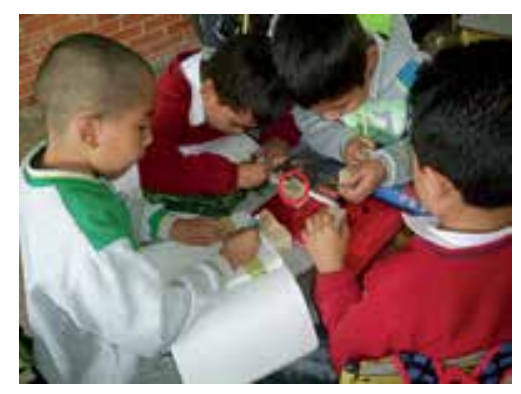

\section{Criterio 1.}

Los estudiantes reconocen los múltiplos de diez de las cintas dadas, y aun sin modelar al metro o la regla en centímetros, los niños miden partes de su alrededor con las cintas y son capaces de expresar la medida en términos de la unidad dispuesta en cintas o partes más pequeñas de la cinta más Salón encintado grande.

\section{Criterio 2.}

Los estudiantes expresan con facilidad, las dimensiones o distancias, además de medir otros objetos, y reconocer a la cinta como un modelo ideal para medir por la facilidad de uso en la
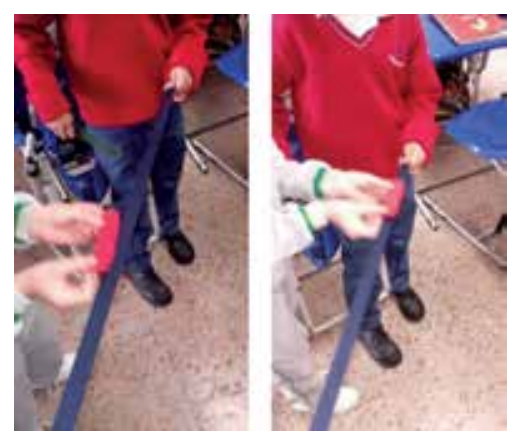
actividad.

\section{Conclusiones}

Un primer resultado, que se exhibe es que en el diseño, gestión y evaluación de la secuencia didáctica, se reconoció la necesidad de crear actividades con criterios enfocados hacia la matematización de situaciones o la modelización de experiencias, pues estas permiten una mayor comprensión por parte de los estudiantes del concepto magnitud-longitud. Así mismo, con la secuencia se identificó una ruta para resolver la primera tensión expuesta.

Un segundo aspecto consiste en que los resultados de la aplicación de las actividades propuestas conducen a modelar las situaciones, que ya fueron matematizadas, haciendo que el proceso de enseñanza-aprendizaje resulte más enriquecedor, en la construcción del concepto magnitud-longitud. Los criterios acerca de las matematizaciones que se vieron implicadas durante todo el proceso fueron realmente evidenciados, y vistos a la luz del referente teórico, de esta manera pudo apreciarse cómo la planeación de estas actividades, fue realmente provechosa para el aprendizaje en el aula de grado segundo, haciendo que la enseñanza-aprendizaje del concepto magnitud-longitud fuera abordado desde otras actividades diferentes a las tradicionales, y que también la actividad de medir resultara una experiencia significativa en cuanto al aprendizaje. 
Un tercer aspecto, nos da una visión de cómo enseñar el concepto magnitud-longitud sin limitarnos solo al uso instruccional de la regla, como único instrumento de medida, pero no permite observar un proceso como el que desarrollamos en la práctica docente, todo esto para llegar al aprendizaje del sistema métrico decimal y por consiguiente la unidad de medida propiamente dicha.

Finalmente, las situaciones matematizables o modelables como las relaciones que nacían a partir de ellas, fueron desarrolladas con éxito, porque dichas relaciones, nos permitían llegar a un desarrollo como el reconocimiento de atributos medibles o la numerosidad de las unidades de medida y nos permitió cubrir bastantes temas que desde una enseñanza tradicional dudosamente se pudieran haber potenciado.

\section{Referencias}

Blomhøj, M. (2004). Mathematical modelling - A theory for practice. International Perspectives on Learning and Teaching Mathematics, 23, 145-159. Traducido por Maria Mina. Recuperado de http://www.famaf. unc.edu.ar/ revm/Volumen23/digital23-2/Modelizacion1.pdf

Chamorro, C., y Belmonte, M. (1994). El problema de la medida. Didáctica de las magnitudes lineales. Madrid: Síntesis.

Freudenthal, H. (1968). Why to teach mathematics as to be useful? Educational Studies in Mathematics, 1(1), 3-8.
Freudenthal, H. (1991). Revisiting Mathematics Education: China Lectures. Dordrecht, The Netherlands: Kluwer.

Gravemeijer, K., y Teruel, J. (2000). Hans Freudenthal, un matemático en didáctica y teoría curricular. J. Currículo Studies, 32, 14.

Grupo DECA. (1992). Orientaciones para el diseño y elaboración de actividades de aprendizaje y de evaluación. Aula, 6, 33-39.

Kieran, C. (1992). The learning and teaching of school algebra. In D. A. Grouws (Ed.), Handbook of research on mathematics teaching and learning (p. 390- 419). New York: Macmillan.

Lurdy, O., Guerrero, F., y Sánchez, N. (2005). La Práctica Docente a Partir del Modelo DECA y La Teoría De Las Situaciones Didácticas. $V$ Festival Internacional de la Matemática. Bogotá.

Niss, M. (1989). Aims and scope of applications and modelling in Mathematics curricula. En W. Blum, y otros (Eds.). Applications and modelling in learning and teaching mathematics (pp. 22-31). Chicester, UK: Horwood Publishing.

Treffers, A., y Goffree, F. (1985). Rational analysis of realistic mathematics education - the Wiskobas program, in L. Streefland (ed.), Proceedings of the Ninth International Conference for the Psychology of Mathematics Education, OW \& OC, Utrecht University, Utrecht, The Netherlands. Treffers, A. (1987). Three Dimensions. A Model of Goal and Theory Description in Mathematics Instruction - The Wiskobas Project. Reidel Publishing Company, Dordrecht, The Netherlands.

Treffers, A. (1991). Didactical background of a mathematics programm for primary education, in L. Streefland (ed.), Realistic Mathematics Education in Primary School, CD-ß Press / Freudenthal Institute, Utrecht University, Utrecht, The Netherlands. 